\title{
Asymmetry of the Active Site Loop Conformation between Subunits of Glutamate-1-semialdehyde Aminomutase in Solution
}

\author{
Barbara Campanini, ${ }^{1}$ Stefano Bettati, ${ }^{2,3}$ Martino Luigi di Salvo, ${ }^{4}$ \\ Andrea Mozzarelli, ${ }^{1,3}$ and Roberto Contestabile ${ }^{4}$ \\ ${ }^{1}$ Dipartimento di Farmacia, Università di Parma, Parco Area delle Scienze 23/A, 43124 Parma, Italy \\ ${ }^{2}$ Dipartimento di Neuroscienze, Università di Parma, Via Volturno 39, 43125 Parma, Italy \\ ${ }^{3}$ Istituto Nazionale di Biostrutture e Biosistemi, Viale Medaglie d'Oro 305, 00136 Roma, Italy \\ ${ }^{4}$ Dipartimento di Scienze Biochimiche "A. Rossi-Fanelli", Sapienza Università di Roma, Piazzale Aldo Moro 5, 00185 Roma, Italy
}

Correspondence should be addressed to Roberto Contestabile; roberto.contestabile@uniromal.it

Received 15 May 2013; Accepted 27 June 2013

Academic Editor: Barbara Cellini

Copyright (c) 2013 Barbara Campanini et al. This is an open access article distributed under the Creative Commons Attribution License, which permits unrestricted use, distribution, and reproduction in any medium, provided the original work is properly cited.

\begin{abstract}
Glutamate-1-semialdehyde aminomutase (GSAM) is a dimeric, pyridoxal $5^{\prime}$-phosphate (PLP)- dependent enzyme catalysing in plants and some bacteria the isomerization of L-glutamate-1-semialdehyde to 5-aminolevulinate, a common precursor of chlorophyll, haem, coenzyme $\mathrm{B}_{12}$, and other tetrapyrrolic compounds. During the catalytic cycle, the coenzyme undergoes conversion from pyridoxamine $5^{\prime}$-phosphate (PMP) to PLP. The entrance of the catalytic site is protected by a loop that is believed to switch from an open to a closed conformation during catalysis. Crystallographic studies indicated that the structure of the mobile loop is related to the form of the cofactor bound to the active site, allowing for asymmetry within the dimer. Since no information on structural and functional asymmetry of the enzyme in solution is available in the literature, we investigated the active site accessibility by determining the cofactor fluorescence quenching of PMP- and PLP-GSAM forms. PLP-GSAM is partially quenched by potassium iodide, suggesting that at least one catalytic site is accessible to the anionic quencher and therefore confirming the asymmetry observed in the crystal structure. Iodide induces release of the cofactor from PMP-GSAM, apparently from only one catalytic site, therefore suggesting an asymmetry also in this form of the enzyme in solution, in contrast with the crystallographic data.
\end{abstract}

\section{Introduction}

Chlorophyll, haem, coenzyme $\mathrm{B}_{12}$, and other tetrapyrrolic compounds share the same biosynthetic origin. They are assembled from four molecules of porphobilinogen, itself the product of the linkage of two 5-aminolevulinate (ALA) molecules [1]. In animals, yeast, and some bacteria, ALA is synthesised in the Shemin pathway, by the condensation of succinyl-CoA with glycine, followed by decarboxylation. The overall reaction is catalysed by ALA synthase, a pyridoxal $5^{\prime}$ phosphate-dependent enzyme [2]. Plants, green algae, and the majority of bacteria synthesise ALA in a completely different way. In these organisms, ALA is obtained through the isomerisation of glutamate-1-semialdehyde (GSA), a compound deriving from glutamate [3]. This reaction is catalysed by glutamate-1-semialdehyde aminomutase (GSAM, EC 5.4.3.8), an enzyme belonging to fold type I PLPdependent enzymes. The primary sequence and tertiary structure of GSAM are strongly related to the class III of aminotransferases [4]. GSAM is a potential target for safe selective herbicides and antibiotics because, while it carries out an essential role in the metabolism of plants and some pathogenic bacteria, it is absent in animals [5].

The mechanism of the aminomutase reaction catalysed by GSAM [6-8] is also closely analogous to that of the transamination reaction [9]. The exceptional feature of GSAM is 
that, unlike aminotransferases, it catalyses an intramolecular exchange of the amino and carbonyl moieties, which are both present in the substrate. The catalytic cycle starts with GSA and the pyridoxamine $5^{\prime}$-phosphate (PMP) form of the enzyme and is completed without the need of a cognate amino acid substrate (Figure 1). A pivotal step in the mechanism is the isomerisation between the two aldimine intermediates that can be formed with either the 4 or the 5-amino group of the 4,5-diaminovalerate (DAVA) intermediate. Whether this part of the reaction takes place through dissociation of DAVA (steps 3 and 4 in Figure 1) or formation of a geminal diamine intermediate (steps $3^{\prime}$ and $4^{\prime}$ in Figure 1) is unclear. Dissociation of DAVA appears mechanistically unnecessary, and even detrimental, although some dissociation has been found to occur to an extent governed by the concentration of the enzyme itself [10]. The PLP form of the enzyme is unable to catalyse the aminomutase reaction [10].

At the end of the purification procedure, dimeric GSAM from Synechococcus contains a mixture of PLP and PMP $[7,11]$. This is invariably observed in GSAM purified from other sources $[8,12]$. Preparations of native GSAM can be treated with substrate analogues that have either an amino or a carbonyl function and, therefore, react in a halftransamination reaction, converting the enzyme into either the PLP form or the PMP form [13]. Notably, crystallographic investigations on native GSAM from Synechococcus showed that the distribution of PLP and PMP was asymmetric within each enzyme dimer $[14,15]$. Moreover, the two subunits showed asymmetry also in the mobility of a 19-residue loop (spanning residues 153-181), which has been shown to control access to the active site and limit the dissociation of the DAVA intermediate [16]. Crystallographic studies have been also carried out on the apoenzyme form and on double PLP or double PMP forms (containing the same form of cofactor in both subunits of the same dimer and obtained by adding either PLP or PMP to the apoenzyme) [15]. In the apo form, the active site loop is disordered in both subunits, whereas in the double PMP form, it is ordered and symmetrically opened. In the double PLP form, one loop is closed and the other is disordered (Table 1). It seems that the loop cannot be present in a closed conformation in both subunits at the same time. It is clear that the conformational state of the loop depends on the particular form of the cofactor present at the active site and is functional to the reaction trajectory of the aminomutase reaction catalysed by GSAM [15, 17]. When PMP is present at the active site, the loop is open, thus allowing substrate entry and product release. In contrast, when the active site contains PLP as internal aldimine and the intermediate DAVA, the loop is closed and access to the active site is obstructed by the short helical section of the loop residues 164-168 [15].

Since no studies on the structural and functional asymmetry of GSAM in solution have been reported in the literature, we investigated the effect of different fluorescence quenchers on the emission properties of the Synechococcus enzyme in solution, in order to ascertain the existence of a relationship between the form of the cofactor present at the active site and its accessibility.

\section{Materials and Methods}

2.1. Materials. GSA aminomutase from Synechococcus was expressed and purified as described previously [11]. All chemicals and buffers were purchased from Sigma-Aldrich (St. Louis, MO, USA) and were of the best available quality.

\subsection{Conversion of the Native Enzyme into the PLP and PMP} Form. Complete conversion of the enzyme into the PLP or PMP form was achieved using succinic semialdehyde and racemic 4, 5-diaminovalerate (DAVA), respectively, and followed by gel filtration. Succinic semialdehyde $(20 \mathrm{mM})$ or DAVA $(5 \mathrm{mM})$ was added to a solution $(1 \mathrm{~mL})$ of enzyme $(60-300 \mu \mathrm{M})$ in $100 \mathrm{mM}$ Na-Tricine buffer, $\mathrm{pH}$ 7.9. Succinic semialdehyde or DAVA in the same buffer $(1 \mathrm{~mL}$, same concentrations) was loaded on a G-25 Sephadex column ( $45 \times$ $1 \mathrm{~cm}$ ) and allowed to drain in before loading the enzyme. The column was equilibrated and eluted with $100 \mathrm{mM} \mathrm{Na}$-Tricine, $\mathrm{pH}$ 7.9. The high molecular mass fraction was collected in the void volume well separated from small molecules.

2.3. Absorbance and Fluorescence Spectroscopy. PLP-GSAM and PMP-GSAM, stored as concentrated solutions in $100 \mathrm{mM}$ Tricine $\mathrm{pH}$ 7.9, were diluted in $20 \mathrm{mM}$ Tricine $\mathrm{pH} 7.9$ for fluorescence and absorption spectroscopy experiments. Experiments were carried out at $25^{\circ} \mathrm{C}$.

Absorption measurements were carried out using a Cary Scan 400 spectrophotometer (Varian Inc., Palo Alto, CA, USA). Temperature was maintained at $25 \pm 0.5^{\circ} \mathrm{C}$ with a circulating water bath.

The fluorescence emission of tryptophans and the four cofactor species under analysis were detected by exciting at $298 \mathrm{~nm}, 320 \mathrm{~nm}, 330 \mathrm{~nm}, 340 \mathrm{~nm}$, and $420 \mathrm{~nm}$, respectively, using a FluoroMax-3 fluorometer (HORIBA Jobin Yvon, Longjumeau Cedex, France) equipped with a thermostated cell holder. Excitation and emission slits were set at $3 \mathrm{~nm}$ except for excitation at $420 \mathrm{~nm}$ where $5 \mathrm{~nm}$ slits were used. Spectra were corrected for buffer contribution. The accessibility of the cofactor was assessed by fluorescence quenching. $\mathrm{CsCl}, \mathrm{KI}$, and acrylamide solutions were prepared in $20 \mathrm{mM}$ Tricine, $\mathrm{pH}$ 7.9. $\mathrm{Na}_{2} \mathrm{~S}_{2} \mathrm{O}_{3}$ at $0.01 \mathrm{mM}$ concentration was added to KI solutions to prevent iodide oxidation. Fluorescence of either $6 \mu \mathrm{M}$ PLP-GSAM or $2 \mu \mathrm{M}$ PMP-GSAM was excited at $330 \mathrm{~nm}$ using $5 \mathrm{~nm}$ slits. Quenching data were analyzed with the Stern-Volmer equation [18]:

$$
\frac{F_{0}}{F}=1+K_{\mathrm{SV}} \cdot[Q]
$$

where $F_{0}$ is the fluorescence intensity in the absence of the quencher, $F$ is the fluorescence at each given quencher concentration, $K_{\mathrm{SV}}$ is the Stern-Volmer constant, and [Q] is the concentration of the quencher.

2.4. Time-Resolved Fluorescence Spectroscopy. Fluorescence intensity decays were measured by the phase and modulation technique $[19,20]$ using an ISS GREG 200 fluorometer (ISS Inc., Champaign, IL, USA). PLP-GSAM fluorescence decays were measured at a protomer concentration of $48.8 \mu \mathrm{M}$, upon 


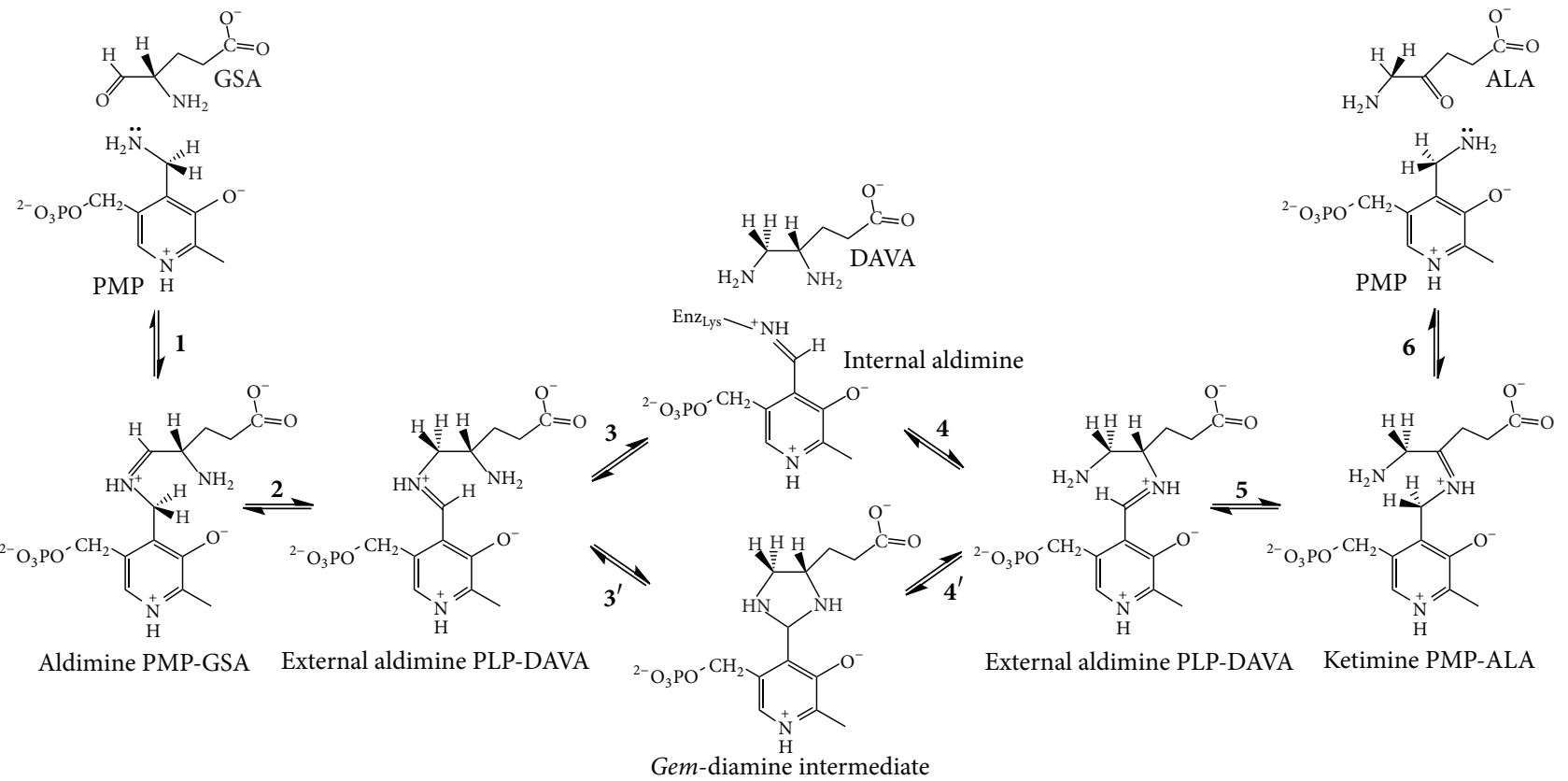

FIGURE 1: Proposed mechanism for the reaction catalysed by GSAM.

TABLE 1: Conformation of the active site loop in different crystal forms of dimeric Synechococcus GSAM [15].

\begin{tabular}{lcc}
\hline Enzyme form & Loop at subunit 1 & Loop at subunit 2 \\
\hline apo-GSAM & Disordered & Disordered \\
PMP-GSAM & Ordered, open & Ordered, open \\
PLP-GSAM & Ordered, closed & Disordered \\
\hline
\end{tabular}

excitation at $330 \mathrm{~nm}$ performed using a $300 \mathrm{~W}$ xenon lamp and a Jobin Yvon monochromator with $16 \mathrm{~nm}$ band width. A p-bis [2-(phenoxazoyl)] benzene (POPOP) solution was used as a lifetime standard reference $(\tau=1.05 \mathrm{~ns})$. To eliminate polarization artifacts in the intensity decay, data were collected under magic angle conditions with the excitation light polarized normal to the laboratory plane, $0^{\circ}$, and the emission polarizer oriented at $54.7^{\circ}$ [20]. Samples were equilibrated at $25 \pm 0.5^{\circ} \mathrm{C}$ using a jacketed cell holder and a circulating water bath. A set of 20 modulation frequencies, varied continuously in the 2 to $200 \mathrm{MHz}$ range, was used. Data were fitted to a sum of discrete exponentials [21] with lifetime $\tau_{i}$ and fractional intensity $f_{i}$ by the Marquardt algorithm of the Globals Unlimited software (University of Illinois, Urbana, IL, USA) [22]. Frequency-independent standard error values of $0.2^{\circ}$ for phase data and 0.004 for modulation data were routinely applied. The $\chi^{2}$ minimization was the criterion used to select the best fits $[19,21]$. The $\tau_{i}$ and $f_{i}$ values reported in Results and Discussion represent the average of three measurements on different samples.

Mean lifetimes $\langle\tau\rangle$ were obtained from lifetimes $\tau_{i}$ and fractional intensities $f_{i}$ according to the equation [23]:

$$
\langle\tau\rangle=\sum \alpha_{i} \cdot \tau_{i}
$$

where $\alpha_{i}$, the preexponential factor for a multiexponential decay law, was determined as

$$
\alpha_{i}=\frac{f_{i} / \tau_{i}}{\sum f_{i} / \tau_{i}} .
$$

\section{Results and Discussion}

3.1. Spectroscopic Properties of PLP-GSAM and PMP-GSAM. Two freshly prepared forms of the enzyme from Synechococcus were used: the PLP form (PLP-GSAM), obtained by treating the native enzyme with succinic-1-semialdehyde, and the PMP form (PMP-GSAM), obtained by the addition of DAVA [13]. The absorption spectra of equimolar solutions of PLP-GSAM and PMP-GSAM are shown in Figure 2. The absorption spectrum of PLP-GSAM shows, in addition to the band of aromatic amino acids centred at $278 \mathrm{~nm}$, a major band centred at $418 \mathrm{~nm}$, typical of the internal aldimine between PLP and the active site lysine, and a modest though measurable absorbance at about $330 \mathrm{~nm}$ that could be attributed to the enolimine tautomer of the internal aldimine or to traces of PMP bound to the active site of the enzyme. Several PLP-binding enzymes exhibit equilibrium between enolimine and ketoenamine tautomers of the Schiff base formed by the coenzyme with the active site lysine (internal aldimine) or with a bound amino acid or reaction intermediate (external aldimines). Ketoenamine tautomers absorb light at longer wavelength with respect to enolimine and are favored in more polar active sites [24]. PMP-GSAM shows a band at $340 \mathrm{~nm}$ attributed to PMP in the active site of the enzyme [6]. No absorption from the internal aldimine of PLP is discernible in the visible range of the spectrum. The absorption spectrum confirms that PMP-GSAM is pure with respect to the content of the cofactor. 


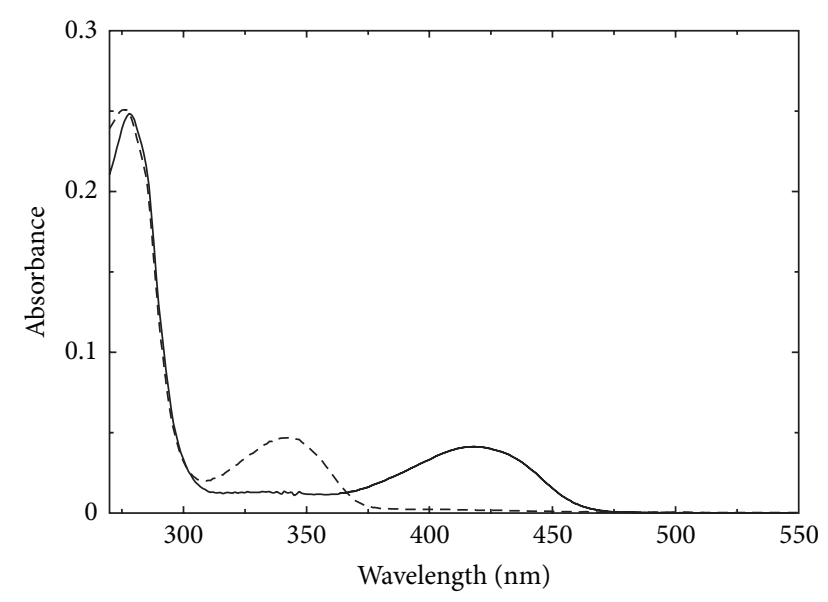

FIgURE 2: Absorption spectra of PLP-GSAM and PMP-GSAM. Absorption spectra of PLP-GSAM (solid line) and PMP-GSAM (dashed line) recorded on a solution containing $7.1 \mu \mathrm{M}$ protein in $20 \mathrm{mM}$ Tricine, $\mathrm{pH} 7.9$.

The intrinsic fluorescence of tryptophans and coenzyme has been previously exploited to investigate structural and dynamic features of some PLP-dependent enzymes and their modification upon binding of substrates, inhibitors, and regulatory molecules [25-30]. In GSAM, both PLP and PMP are fluorescent upon excitation in either the UV or visible range of the spectrum. At $298 \mathrm{~nm}$, tryptophan fluorescence is selectively excited, with almost no contribution from the other aromatic side chains of tyrosine and phenylalanine. When excited at $298 \mathrm{~nm}$, both PLP-GSAM and PMP-GSAM show an emission band centred at about $345 \mathrm{~nm}$ characteristic of direct tryptophan emission (Figure 3(a)). Emission from PMP-GSAM is slightly less intense than that of PLPGSAM. GSAM has two tryptophans, Trp53 and Trp67. Trp67 is buried inside the protein structure at about $10 \AA$ from the cofactor, whereas Trp53 is exposed to the solvent far away from the active site. The distance between Trp67 and the coenzyme is compatible with Förster resonance energy transfer from the former to the latter [25, 31]. However, differently from what observed for other PLP-dependent enzymes like $O$-acetylserine sulfhydrylase [25] or tryptophan synthase [32], the perpendicular spatial disposition of the two chromophores appears to hinder an efficient energy transfer, as demonstrated by the emission spectrum upon excitation at $298 \mathrm{~nm}$ where no bands around 500, attributable to coenzyme emission, are observed, except for a barely detectable shoulder in the case PLP-GSAM (Figure 3(a)).

Upon excitation at $420 \mathrm{~nm}$, only PLP-GSAM yields a significant emission, with a band centered at $500 \mathrm{~nm}$ (Figure 3(b)). In agreement with absorption data, this observation further supports the notion that PMP-GSAM samples do not carry significant amounts of cofactor in the PLP form.

Both PMP-GSAM and PLP-GSAM show fluorescence emission upon excitation at around $330 \mathrm{~nm}$ (Figures 3(c) and $3(\mathrm{~d}))$. The shape of PMP-GSAM emission spectrum does not depend on the excitation wavelength in the 320$340 \mathrm{~nm}$ range, and the emission intensity is maximum at $\lambda_{\mathrm{ex}}=340 \mathrm{~nm}$, consistent with the absorbance spectrum (Figure 2). The emission spectra of PLP-GSAM for excitation at 320,330 , and $340 \mathrm{~nm}$ also show a broad band centred at about $390 \mathrm{~nm}$, that could be due to the direct emission of the enolimine tautomer of PLP or to the presence of some fraction of subunits carrying a chromophore in the PMP form. If the latter was predominantly the case, we would expect fluorescence emission to parallel the behaviour of PMP-GSAM, with emission intensity increasing consistently from $\lambda_{\mathrm{ex}}=320 \mathrm{~nm}$ to $\lambda_{\mathrm{ex}}=340 \mathrm{~nm}$. Moreover, the emission band of PLP-GSAM excited at 320 and $330 \mathrm{~nm}$ appears to have a slightly red-shifted (by 3-4 nm) peak wavelength with respect to PMP-GSAM, and is definitely more asymmetric, being broader on the low energy side of the emission spectrum (the ratio of emission intensity at 390 and $500 \mathrm{~nm}$ is about 5, compared to more than 20 for PMPGSAM). Furthermore, differently from PMP-GSAM, when PLP-GSAM is excited at $340 \mathrm{~nm}$, the main emission band is slightly decreased in intensity and is accompanied by a shoulder at about $500 \mathrm{~nm}$ (Figure 3(c)), most likely due to direct excitation of the high energy tail of the absorption band of PLP ketoenamine tautomer [30, 33, 34]. The presence of a discrete emission at around $500 \mathrm{~nm}$ could also originate from the ketoenamine tautomer that forms in the excited state following proton transfer from the $3^{\prime}-\mathrm{OH}$ group to the imine nitrogen $[35,36]$. This observation further supports the view that the absorption at around $330 \mathrm{~nm}$ arises from the enolimine tautomer of the internal aldimine rather than from a substituted aldimine, as previously pointed out for other PLP-dependent enzymes [35, 37].

The results of absorbance and fluorescence emission spectroscopic characterization clearly indicate that the PLPGSAM and PMP-GSAM preparations used in this work are at least $90 \%$ pure with respect to the state of the bound cofactor.

3.2. Fluorescence Quenching. Fluorescence quenching is a powerful technique to assess the accessibility of fluorophores to the solvent. We have previously exploited this approach to investigate changes in cofactor accessibility of wild-type and mutant forms of another PLP-dependent enzyme, Oacetylserine sulfhydrylase [38]. Quenchers that are commonly used in protein fluorescence are caesium (as chloride salt), iodide (as potassium salt), and acrylamide. The different polarity and charge of these molecules allow the application of fluorescence quenching to assess the accessibility of both apolar and polar sites. In fact, the polarity of the site entrance influences the access of the quencher independently of the steric accessibility of the site. The active site of GSAM is mainly edged by positively charged residues likely to hamper the access to the positively charged caesium ions [14]. Quenching experiments were carried out exciting the fluorescence of the cofactor at $330 \mathrm{~nm}$. Both acrylamide and caesium chloride proved to be ineffective at quenching the cofactor fluorescence. In particular, whereas acrylamide does not show any effect on the emission intensity of the cofactor up to about $1 \mathrm{M}$, caesium chloride induces an increase, rather than a decrease, in the cofactor emission intensity of both PMP-GSAM and PLP-GSAM (Figure 4(a)). The same effect 


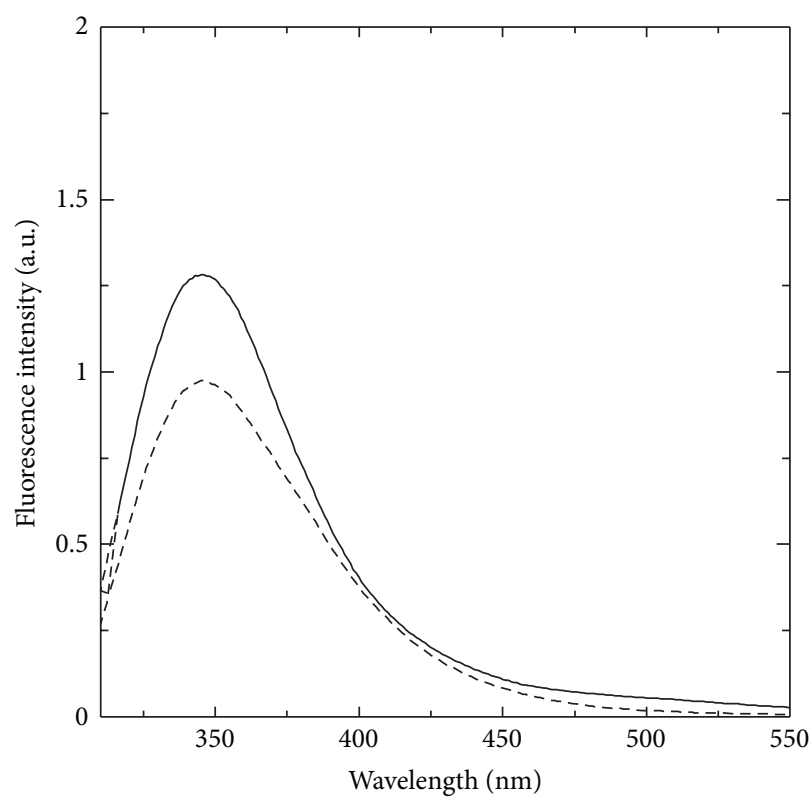

(a)

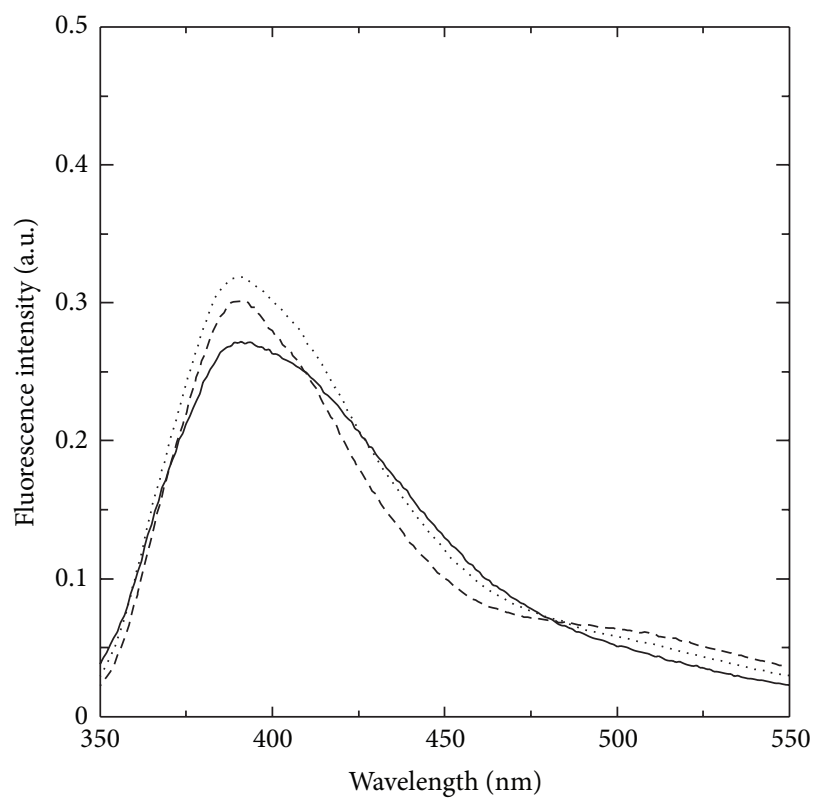

(c)

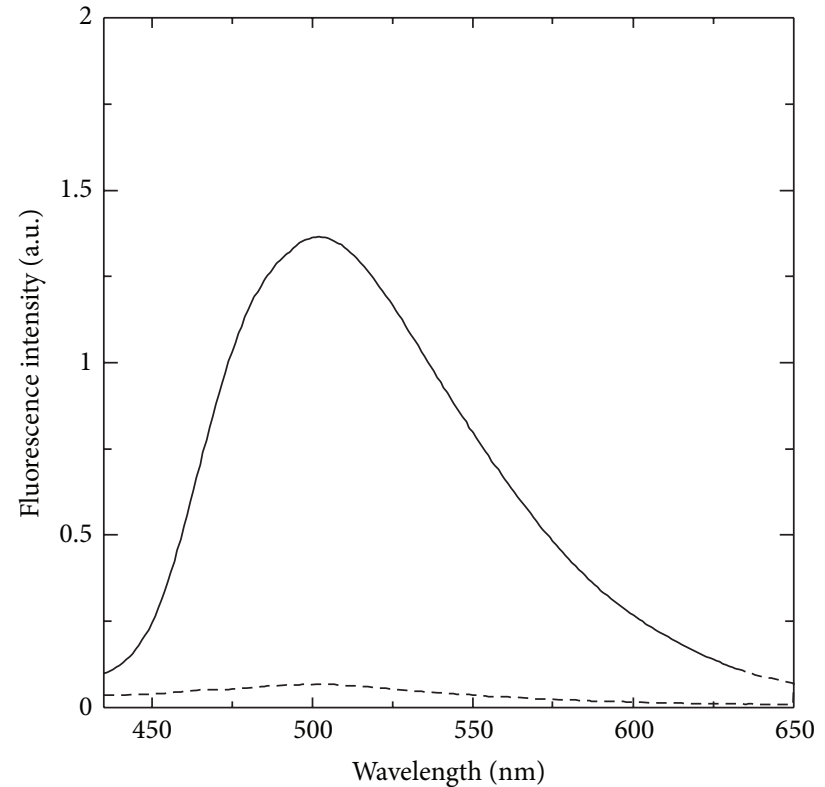

(b)

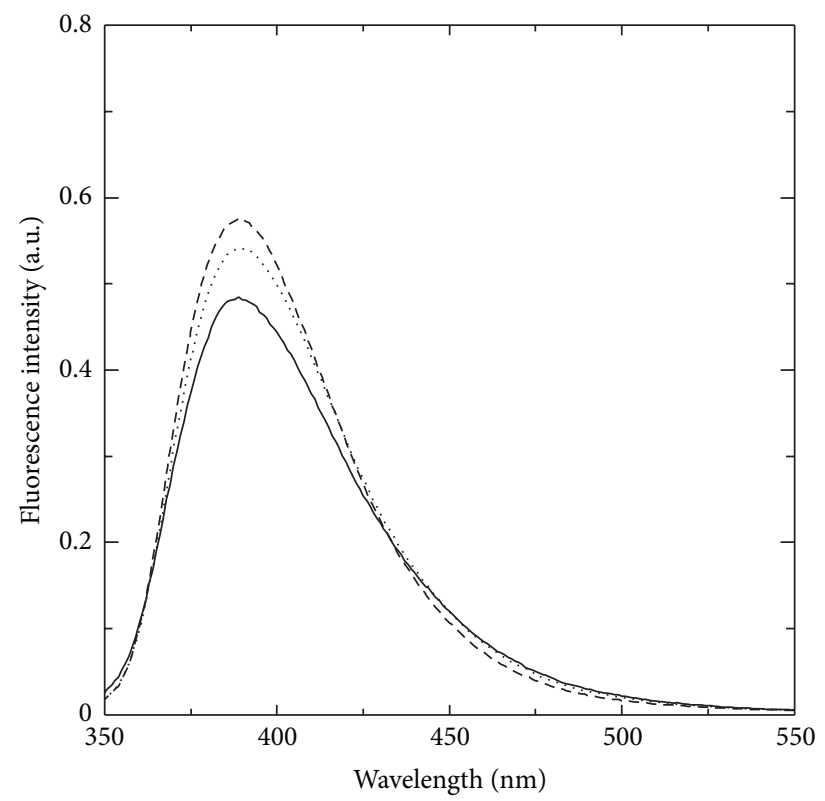

(d)

Figure 3: Fluorescence emission spectra of PLP-GSAM and PMP-GSAM. To allow direct comparison of the data, the reported spectra are normalized for protein concentration. Spectra were collected in $20 \mathrm{mM}$ Tricine buffer, $\mathrm{pH} 7.9$ solutions and were normalized to a protein concentration of $7 \mu \mathrm{M}$. (a) Emission spectra of PLP-GSAM (solid line) and PMP-GSAM (dashed line) upon excitation at $298 \mathrm{~nm}$ with $3 \mathrm{~nm}$ emission and excitation slits. (b) Emission spectra of PLP-GSAM (solid line) and PMP-GSAM (dashed line) upon excitation at $420 \mathrm{~nm}$ with $5 \mathrm{~nm}$ emission and excitation slits. (c) Emission spectra of PLP-GSAM upon excitation at $320 \mathrm{~nm}$ (solid line), $330 \mathrm{~nm}$ (dotted line), and $340 \mathrm{~nm}$ (dashed line) with $3 \mathrm{~nm}$ emission and excitation slits. (d) Emission spectra of PMP-GSAM upon excitation at $320 \mathrm{~nm}$ (solid line), $330 \mathrm{~nm}$ (dotted line), and $340 \mathrm{~nm}$ (dashed line) with $3 \mathrm{~nm}$ emission and excitation slits.

is observed if caesium chloride is substituted by potassium chloride (Figure 4(a)) or potassium acetate (data not shown), suggesting a nonspecific effect of the ionic strength on the fluorescence quantum yield of the cofactor. The effect is more pronounced on PMP-GSAM, leading to a doubling of the signal at about $140 \mathrm{mM}$ salt.
On the other hand, potassium iodide is effective in quenching the fluorescence of PLP-GSAM (Figure 4(b)), indicating that at least one catalytic site is in an open conformation, accessible to the anionic quencher. The linearity of data shown in Figure 4(b) demonstrates that the enolimine form of enzyme-bound PLP is exposed to a structurally 


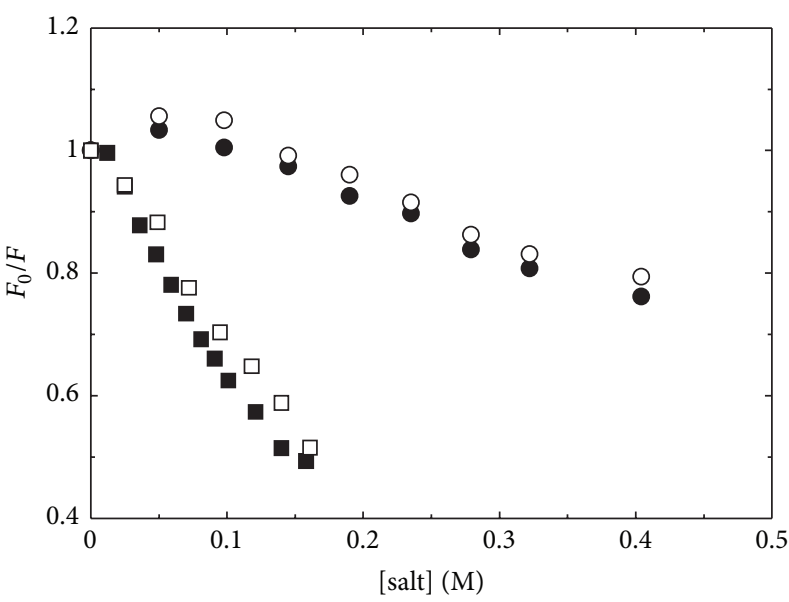

(a)

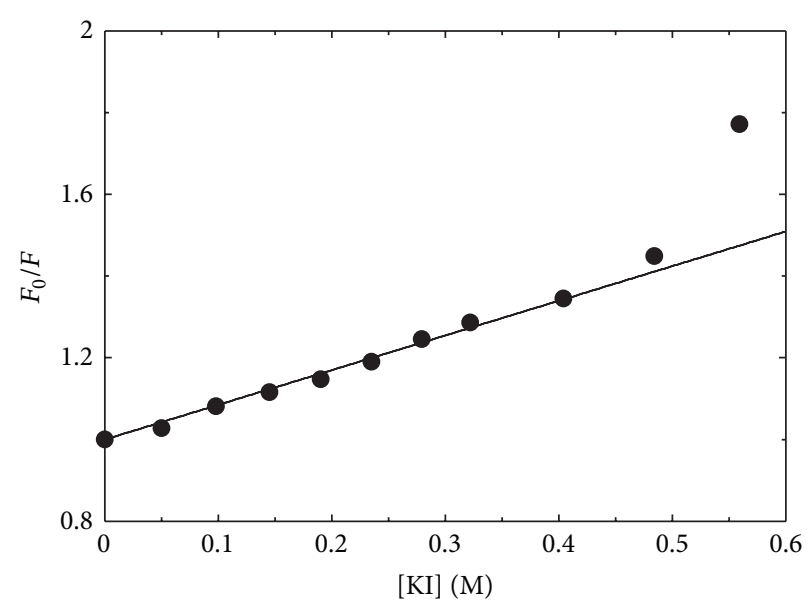

(b)

FIGURE 4: Dependence of fluorescence emission intensity on quencher concentration. (a) Dependence of the relative emission intensity at $384 \mathrm{~nm}$, upon excitation at $330 \mathrm{~nm}$, of PLP-GSAM (circles) and PMP-GSAM (squares) on caesium chloride (closed symbols) and potassium chloride (open symbols) concentration. (b) Stern-Volmer plot for the quenching of PLP-GSAM by iodide. The line through data points represents the fitting to (1).

homogeneous environment. The ionic radius of $\mathrm{Cs}^{+}$is about $1.6 \AA$ whereas the ionic radius of $\mathrm{I}^{-}$is about $2.2 \AA$, so the differences in the accessibility to the active site cannot be ascribed to steric effects but rather to electrostatic repulsion between $\mathrm{Cs}^{+}$and polar or positively charged groups at the active site entrance. The analysis of experimental data in Figure 4(b) according to (1) (Materials and Methods) gave a Stern-Volmer constant $\left(K_{\mathrm{SV}}\right)$ value of $0.84 \pm 0.01 \mathrm{M}^{-1}$. Since the value of $K_{\mathrm{SV}}$ that we measured for quenching by iodide of free PLP in solution is $2.61 \pm 0.07 \mathrm{M}^{-1}$, in agreement with data present in the literature [39], it appears that the active site of PLP-GSAM is only partially accessible to the solvent. More significantly, the difference between the bimolecular quenching rate constants (i.e., the ratio between $K_{\mathrm{SV}}$ and the lifetime of the excited state [39-41]) of free PLP and PLPGSAM complexes, that are the true measure of active site accessibility, should be larger than the difference between the Stern-Volmer constants, considering that the fluorescence lifetime of free PLP is expected to be significantly smaller than that of protein-bound PLP. The fluorescence lifetime decays of PLP-GSAM are well fitted by two discrete exponential components with lifetimes of $8.6 \pm 0.2$ and $1.40 \pm 0.05 \mathrm{~ns}$ and fractional intensity of $10.3 \pm 0.8 \%$ and $89.7 \pm 0.8 \%$, respectively, (data not shown). The mean lifetime, calculated as reported in Materials and Methods, is $1.53 \mathrm{~ns}$. This value can be compared to those calculated from data reported in the literature [25] for free PLP in solution and PLP-Lvaline, a model compound of PLP Schiff base, upon excitation at $330 \mathrm{~nm}: 0.32 \mathrm{~ns}$ and $0.68 \mathrm{~ns}$, respectively. By considering the latter a better mimic of PLP bound to the enzyme as an internal aldimine, a ratio of about 7 can be calculated between the apparent bimolecular quenching rate constant of PLP-L-valine $\left(3.84 \cdot 10^{-9} \mathrm{M}^{-1} \mathrm{~s}^{-1}\right)$ and PLP-GSAM $(0.55$. $10^{-9} \mathrm{M}^{-1} \mathrm{~s}^{-1}$ ). These values are in good agreement with those previously reported for free coenzyme and the internal aldimine of the PLP-dependent enzyme tryptophan synthase $\alpha_{2} \beta_{2}$ complex $\left(0.52 \cdot 10^{-9} \mathrm{M}^{-1} \mathrm{~s}^{-1}\right)$ [39], known to be in an "open" conformation [42].

At potassium iodide concentrations higher than $0.5 \mathrm{M}$, the Stern-Volmer plot of PLP-GSAM quenching by iodide shows an upward curvature (Figure 4(b)) that could be due to a static quenching component or to a conformational change in the protein structure that leads to a change in the accessibility of the active site to the quencher. For example, an upward curvature of the Stern-Volmer plot of the tetrameric PLP-dependent enzyme tryptophan synthase was attributed to subunit dissociation induced by potassium iodide [39].

Interestingly, the same iodide quenching experiment on PLP-GSAM, carried out following the fluorescence emission intensity upon excitation at $420 \mathrm{~nm}$, gives only small changes in the relative fluorescence intensity (data not shown). This suggests that the fraction of PLP present in the ketoenamine form is less accessible by the anionic quencher. This could be due in principle to a closed conformation of the active site or to a different arrangement of polar and charged residues at the active entrance with respect to the enolimine tautomer.

All together, the results obtained with PLP-GSAM indicate, in agreement with the crystallographic data, a degree of structural asymmetry in the microenvironment surrounding the enzyme-bound PLP. This asymmetry might correspond to an asymmetric distribution of the ketoenamine (less accessible) and enolimine forms of PLP at the two active sites of the enzyme.

The dependence of the emission spectrum of PMPGSAM on potassium iodide concentration is complex. The intensity of the emission upon excitation at $330 \mathrm{~nm}$ increases with increasing KI concentrations up to about $250 \mathrm{mM}$ and then decreases with a red shift of the peak maximum (data not shown). Differently from what we observed on PLP-GSAM, the emission signal in the presence of KI is not stable with 


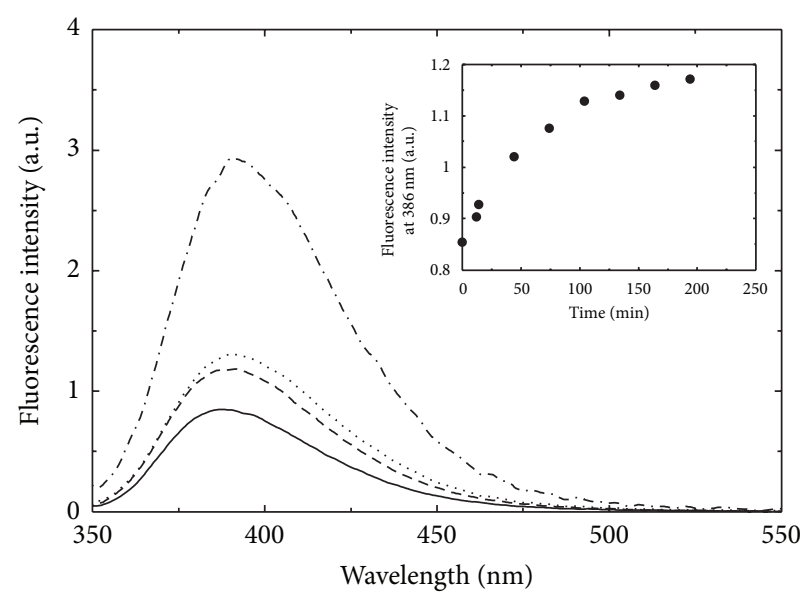

(a)

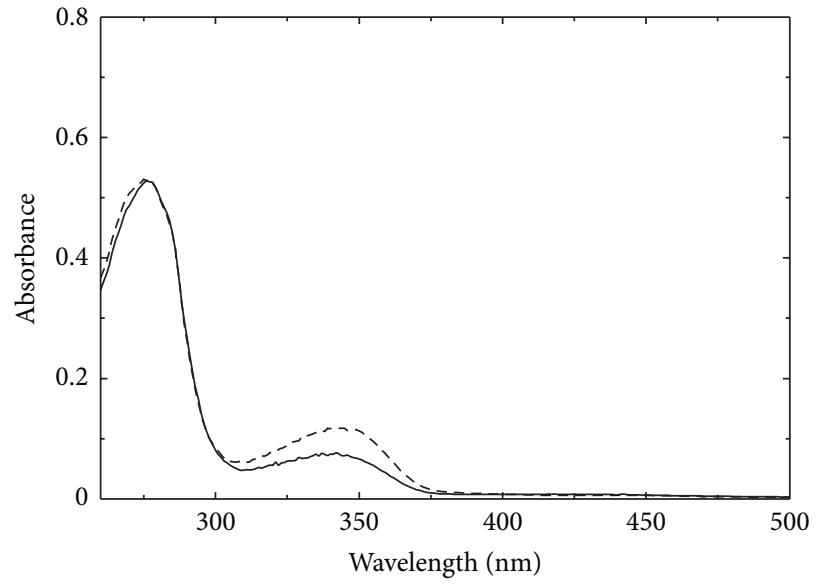

(b)

FiguRE 5: KI-induced release of the cofactor from PMP-GSAM. (a) Fluorescence emission spectra upon excitation at $330 \mathrm{~nm}$ of $2 \mu \mathrm{M}$ PMPGSAM in the absence (solid line) and in the presence (dashed line) of $50 \mathrm{mM} \mathrm{KI}$ (after 195 minutes incubation). The emission spectra of $2 \mu \mathrm{M}$ PMP in Tricine (dashed-dotted line) and $1 \mu \mathrm{M}$ PMP in tricine plus $1 \mu \mathrm{M}$ PMP-GSAM (dotted line) are shown for comparison. Inset: time course of the fluorescence emission intensity of $2 \mu \mathrm{M}$ PMP-GSAM $\left(\lambda_{\mathrm{ex}}=330 \mathrm{~nm}, \lambda_{\mathrm{em}}=386 \mathrm{~nm}\right)$ after the addition of $50 \mathrm{mM} \mathrm{KI}$. (b) Absorbance spectrum of a solution of PMP-GSAM before (solid line) and after (dashed line) treatment with 50 mM KI. The protein solution was mixed with a concentrated solution of KI to a final concentration of $50 \mathrm{mM}$ and extensively dialyzed first against $50 \mathrm{mM}$ KI in tricine buffer $\mathrm{pH} 7.9$ and then against KI-free buffer.

time, but, at low KI concentrations, it increases with time (Figure 5(a), inset). The fluorescence emission intensity of PMP free in solution is about 3 times higher than that of PMP bound to GSAM (Figure 5(a)). The increase of fluorescence emission caused by KI could thus be due to the release of the cofactor from the catalytic site, and the decrease and red-shifting of emission intensity at higher quencher concentration might be related to quenching of PMP free in solution. Indeed, the spectrum of $2 \mu \mathrm{M}$ PMP-GSAM after 195 minutes incubation in $50 \mathrm{mM}$ KI nearly coincides with that calculated, adding the absorption of $1 \mu \mathrm{M}$ PMP in the presence of $50 \mathrm{mM}$ KI to that of $1 \mu \mathrm{M}$ PMP-GSAM. This finding suggests that about $50 \%$ of the cofactor has been released from PMP-GSAM complex. This was confirmed by dialysis experiments in which a solution of PMP-GSAM containing $50 \mathrm{mM}$ KI was first dialysed against $50 \mathrm{mM} \mathrm{KI}$ and then against KI-free buffer. After dialysis, the absorption peak of the cofactor was reduced by 1.6-fold (Figure 5(b)), further indicating that treatment with KI causes the release of about 50\% PMP from PMP-GSAM. Other anions were previously shown to cause, at high concentration, the release of PMP from the active site, for example, GSAM from pea leaves treated with $0.2 \mathrm{M}$ phosphate at acidic $\mathrm{pH}$ [43]. Interestingly, also in that case the release of PMP was not complete, about $44 \%$ of the theoretical amount.

Our results, showing that 50\% of PMP dissociates from the enzyme at low KI concentration, suggest the release of PMP from only one active site and imply a structural asymmetry of the double PMP form of GSAM. This is in contrast with the previously published crystallographic data, showing that in the PMP-GSAM form, the active site loop is ordered and symmetrically opened in both subunits [15]. The asymmetry of PMP-GSAM in solution could be either preexisting or be generated once a single PMP molecule has left the dimer. The preexisting asymmetry could originate from the different experimental protocols used to prepare PMP-GSAM: by addition of PMP to the apoenzyme (crystallographic data), or by the addition of DAVA to the native enzyme, that is typically purified as an asymmetric PLP/PMP form (this work). In our PMP-GSAM, one active site of the dimer may still contain the reaction product. Indeed, we previously observed some instability, upon prolonged storage, of the PLP- and PMP-GSAM preparations obtained by reacting native GSAM with substrate analogues undergoing half-transamination reaction. Although from the absorption spectra, they appear to contain only one or the other form of the cofactor when freshly prepared, they seem to slowly revert to a form containing both PLP and PMP (data not shown). Interestingly, in the characterisation of different spectral forms of GSAM by electrospray mass spectrometry, Brody et al. [44] reported that reduction with $\mathrm{NaBH}_{4}$ of the PLP form yielded two molecular species of different mass, suggesting that the cofactor was actually present also as PMP. The PMP form obtained by the same authors was also recognised to have a "barely detectable" absorption peak at $420 \mathrm{~nm}$.

\section{Conclusion}

The asymmetry of the active site loop conformation between subunits of dimeric glutamate-1-semialdehyde aminomutase in solution was investigated by fluorescence spectroscopy. Cationic and neutral quenchers are ineffective in quenching PMP-GSAM and PLP-GSAM fluorescence, likely due to the distribution of positive charges that characterizes the entrance of the active site of both enzyme forms. On the other 
hand, KI quenches PLP-GSAM fluorescence, although only the fraction of coenzyme molecules present as the enolimine tautomer is significantly accessible to the solvent, clearly indicating an asymmetric structure of the active sites. These observations match the crystallographic data, which show an asymmetric conformation of the loop controlling access to the active site, so that when the enzyme is in the double PLP form, one loop is closed and the other is disordered [15]. Due to the known effect of environment polarity on the equilibrium between the enolimine and ketoenamine forms of PLP, it is tempting to assume that selective quenching of the enolimine arises from asymmetric loop closure stabilizing a different equilibrium of tautomers at the two active sites.

Comparatively low concentrations of KI cause the release of PMP from PMP-GSAM, apparently only from one subunit of the dimer. A possible interpretation of this result is that the double PMP-GSAM presents active site structural asymmetry, in contrast to previous crystallographic reports. Alternatively, this asymmetry may be generated once one PMP molecule has left the dimeric enzyme. This latter explanation is not in contrast with the crystallographic data and relies on the fact that communication between subunits of the GSAM dimer has been shown experimentally and has been proposed on the basis of structural and mechanistic considerations [15-17].

Other important examples of involvement of an active site gating loop in PLP-dependent catalysis are found in foldtype I decarboxylases, such as human isoforms of glutamate decarboxylase [45] and DOPA decarboxylase [46]. In these enzymes, an active site loop plays a pivotal role in controlling reaction specificity. In $O$-acetylserine sulfhydrylase, the interaction of the substrate carboxylate with a substrate-binding loop, highly conserved from bacteria to plants, triggers the closure of the active site to favor catalysis through proper positioning of substrate-binding groups and exclusion of water molecules [47-49]. Indeed, the control of substrate and reaction specificity, and catalytic efficiency, through an equilibrium between "open" and "closed" states of the active site appears to be a common feature in PLP-dependent enzymes [50].

\section{Abbreviations}

$\begin{array}{ll}\text { ALA: } & \text { 5-Aminolevulinate } \\ \text { DAVA: } & \text { 4,5-Diaminovalerate } \\ \text { GSA: } & \text { L-Glutamate-1-semialdehyde } \\ \text { GSAM: } & \text { Glutamate-1-semialdehyde aminomutase } \\ \text { PLP: } & \text { Pyridoxal 5' } \text {-phosphate } \\ \text { PLP-GSAM: } & \text { GSAM form with PLP bound to both } \\ & \text { subunits of the dimer } \\ \text { PMP: } & \text { Pyridoxamine 5' } \text {-phosphate } \\ \text { PMP-GSAM: } & \text { GSAM form with PMP bound to both } \\ & \text { subunits of the dimer. }\end{array}$

\section{Acknowledgments}

This work was partly supported by grants from the Italian Ministero dell'Istruzione, dell'Università e della Ricerca and from Finanziamento Progetti di Ricerca 2011 of Sapienza University of Rome.

\section{References}

[1] P. M. Jordan and D. Shemin, $\delta$-Aminolevulinic Acid Synthetase, in the Enzymes, Academic Press, New York, NY, USA, 1972.

[2] S. I. Beale and J. D. Weinstein, Biosynthesis of Haem and Chlorophylls, McGraw-Hill, New York, NY, USA, 1990.

[3] C. G. Kannangara, S. P. Gough, P. Bruyant, J. K. Hoober, A. Kahn, and D. von Wettstein, "tRNA(Glu) as a cofactor in deltaaminolevulinate biosynthesis: steps that regulate chlorophyll synthesis," Trends in Biochemical Sciences, vol. 13, pp. 139-143, 1988.

[4] N. V. Grishin, M. A. Phillips, and E. J. Goldsmith, "Modeling of the spatial structure of eukaryotic ornithine decarboxylases," Protein Science, vol. 4, pp. 1291-1304, 1995.

[5] R. Contestabile, T. Jenn, M. Akhtar, D. Gani, and R. A. John, "Reactions of glutamate 1-semialdehyde aminomutase with Rand S-enantiomers of a novel, mechanism-based inhibitor, 2,3diaminopropyl sulfate," Biochemistry, vol. 39, no. 11, pp. 30913096, 2000.

[6] M. A. Smith, C. G. Kannangara, B. Grimm, and D. Von Wettstein, "Characterization of glutamate-1-semialdehyde aminotransferase of Synechococcus. Steady-state kinetic analysis," European Journal of Biochemistry, vol. 202, no. 3, pp. 749-757, 1991.

[7] M. A. Smith, B. Grimm, C. G. Kannangara, and D. Von Wettstein, "Spectral kinetics of glutamate-1-semialdehyde aminomutase of Synechococcus," Proceedings of the National Academy of Sciences of the United States of America, vol. 88, no. 21, pp. 9775-9779, 1991.

[8] C. E. Pugh, J. L. Harwood, and R. A. John, "Mechanism of glutamate semialdehyde aminotransferase: roles of diaminoand dioxo-intermediates in the synthesis of aminolevulinate," Journal of Biological Chemistry, vol. 267, no. 3, pp. 1584-1588, 1992.

[9] P. Christen and D. E. Metzler, Transaminases, Wiley, New York, NY, USA, 1995.

[10] R. J. Tyacke, J. L. Harwood, and R. A. John, "Properties of the pyridoxaldimine form of glutamate semialdehyde aminotransferase (glutamate-1-semialdehyde 2,1-aminomutase) and analysis of its role as an intermediate in the formation of aminolaevulinate," Biochemical Journal, vol. 293, part 3, pp. 697701, 1993.

[11] B. Grimm, A. J. Smith, C. G. Kannangara, and M. Smith, "Gabaculine-resistant glutamate 1-semialdehyde aminotransferase of Synechococcus: deletion of a tripeptide close to the $\mathrm{NH} 2$ terminus and internal amino acid substitution," Journal of Biological Chemistry, vol. 266, no. 19, pp. 12495-12501, 1991.

[12] L. L. Ilag and D. Jahn, "Activity and spectroscopic properties of the Escherichia coli glutamate 1-semialdehyde aminotransferase and the putative active site mutant K265R," Biochemistry, vol. 31, no. 31, pp. 7143-7151, 1992.

[13] S. D’Aguanno, I. N. Gonzales, M. Simmaco, R. Contestabile, and R. A. John, "Stereochemistry of the reactions of glutamate-1semialdehyde aminomutase with 4,5-diaminovalerate," Journal of Biological Chemistry, vol. 278, no. 42, pp. 40521-40526, 2003.

[14] M. Hennig, B. Grimm, R. Contestabile, R. A. John, and J. N. Jansonius, "Crystal structure of glutamate-1-semialdehyde aminomutase: an $\alpha 2$-dimeric vitamin B6-dependent enzyme 
with asymmetry in structure and active site reactivity," Proceedings of the National Academy of Sciences of the United States of America, vol. 94, no. 10, pp. 4866-4871, 1997.

[15] J. Stetefeld, M. Jenny, and P. Burkhard, "Intersubunit signaling in glutamate-1-semialdehyde-aminomutase," Proceedings of the National Academy of Sciences of the United States of America, vol. 103, no. 37, pp. 13688-13693, 2006.

[16] R. Contestabile, S. Angelaccio, R. Maytum, F. Bossa, and R. A. John, "The contribution of a conformationally mobile, active site loop to the reaction catalyzed by glutamate semialdehyde aminomutase," Journal of Biological Chemistry, vol. 275, no. 6, pp. 3879-3886, 2000.

[17] J. L. Sorensen and J. Stetefeld, "Kinemage of action-proposed reaction mechanism of glutamate-1-semialdehyde aminomutase at an atomic level," Biochemical and Biophysical Research Communications, vol. 413, no. 4, pp. 572-576, 2011.

[18] M. R. Eftink and C. A. Ghiron, "Fluorescence quenching studies with proteins," Analytical Biochemistry, vol. 114, no. 2, pp. 199227, 1981.

[19] E. Gratton and M. Limkeman, "A continuously variable frequency cross-correlation phase fluorometer with picosecond resolution," Biophysical Journal, vol. 44, no. 3, pp. 315-324, 1983.

[20] R. D. Spencer and G. Weber, "Measurements of subnanosecond fluorescence lifetimes with a cross-correlation phase fluorometer," Annals of the New York Academy of Sciences, vol. 158, pp. 361-376, 1969.

[21] D. M. Jameson and T. L. Hazlett, "Time resolved fluorescence in biology and biochemistry," in Biophysical and Biochemical Aspects of Fluorescence Spectroscopy, T. G. Dewey, Ed., pp. 106133, Plenum, New York, NY, USA, 1991.

[22] J. M. Beechem and E. Gratton, "Time-resolved laser spectroscopy in biochemistry," in Proceedings of the SPIE, International Society for Optical Engineering, Bellingham, Wash, USA, 1988.

[23] M. R. Eftink, "The use of fluorescence methods to monitor unfolding transitions in proteins," Biophysical Journal, vol. 66, no. 2 I, pp. 482-501, 1994.

[24] E. J. Faeder and G. G. Hammes, "Kinetic studies of tryptophan synthetase. Interaction of L-serine, indole, and tryptophan with the native enzyme," Biochemistry, vol. 10, no. 6, pp. 1041-1045, 1971.

[25] S. Benci, S. Vaccari, A. Mozzarelli, and P. F. Cook, "Timeresolved fluorescence of $O$-acetylserine sulfhydrylase," Biochimica et Biophysica Acta, vol. 1429, no. 2, pp. 317-330, 1999.

[26] E. Salsi, R. Guan, B. Campanini et al., "Exploring $O$-acetylserine sulfhydrylase-B isoenzyme from Salmonella typhimurium by fluorescence spectroscopy," Archives of Biochemistry and Biophysics, vol. 505, no. 2, pp. 178-185, 2011.

[27] A. Mozzarelli and S. Bettati, "Exploring the pyridoxal $5^{\prime}$ phosphate-dependent enzymes," Chemical Record, vol. 6, no. 5, pp. 275-287, 2006.

[28] A. Chattopadhyay, M. Meier, S. Ivaninskii et al., "Structure, mechanism, and conformational dynamics of $\mathrm{O}$-acetylserine sulfhydrylase from Salmonella typhimurium: comparison of A and B isozymes," Biochemistry, vol. 46, no. 28 , pp. $8315-8330$, 2007.

[29] E. Salsi, B. Campanini, S. Bettati et al., "A two-step process controls the formation of the bienzyme cysteine synthase complex," Journal of Biological Chemistry, vol. 285, no. 17, pp. 12813-12822, 2010.
[30] E. Pennacchietti, T. M. Lammens, G. Capitani et al., "Mutation of His465 alters the $\mathrm{pH}$-dependent spectroscopic properties of Escherichia coli glutamate decarboxylase and broadens the range of its activity toward more alkaline $\mathrm{pH}$," Journal of Biological Chemistry, vol. 284, no. 46, pp. 31587-31596, 2009.

[31] P. J. R. Lakowicz, Principles of Fluorescence Spectroscopy, Plenum Press, New York, NY, USA, 1983.

[32] S. Vaccari, S. Benci, A. Peracchi, and A. Mozzarelli, "Timeresolved fluorescence of tryptophan synthase," Biophysical Chemistry, vol. 61, no. 1, pp. 9-22, 1996.

[33] M. Arrio-Dupont, "Fluorescence study of Schiff bases of pyridoxal. Comparison with L-aspartate aminotransferase," Photochemistry and Photobiology, vol. 12, no. 4, pp. 297-315, 1970.

[34] E. Passera, B. Campanini, F. Rossi et al., "Human kynurenine aminotransferase II-reactivity with substrates and inhibitors," FEBS Journal, vol. 278, no. 11, pp. 1882-1900, 2011.

[35] M. T. Olmo, F. Sánchez-Jiménez, M. A. Medina, and H. Hayashi, "Spectroscopic analysis of recombinant rat histidine decarboxylase," Journal of Biochemistry, vol. 132, no. 3, pp. 433439, 2002.

[36] S. Benci, S. Vaccari, A. Mozzarelli, and P. F. Cook, "Timeresolved fluorescence of $O$-acetylserine sulfhydrylase catalytic intermediates," Biochemistry, vol. 36, no. 49, pp. 15419-15427, 1997.

[37] M. Bertoldi, B. Cellini, T. Clausen, and C. B. Voltattorni, "Spectroscopic and kinetic analyses reveal the pyridoxal $5^{\prime}$-phosphate binding mode and the catalytic features of Treponema denticola cystalysin," Biochemistry, vol. 41, no. 29, pp. 9153-9164, 2002.

[38] B. Campanini, S. Raboni, S. Vaccari et al., "Surface-exposed tryptophan residues are essential for $O$-acetylserine sulfhydrylase structure, function, and stability," Journal of Biological Chemistry, vol. 278, no. 39, pp. 37511-37519, 2003.

[39] A. N. Lane, "The accessibility of the active site and conformation states of the beta 2 subunit of tryptophan synthase studied by fluorescence quenching," European Journal of Biochemistry, vol. 133, no. 3, pp. 531-538, 1983.

[40] M. R. Eftink and C. A. Ghiron, "Exposure of tryptophanyl residues in proteins. Quantitative determination by fluorescence quenching studies," Biochemistry, vol. 15, no. 3, pp. 672680, 1976.

[41] M. R. Eftink and C. A. Ghiron, "Fluorescence quenching of indole and model micelle systems," Journal of Physical Chemistry, vol. 80, no. 5, pp. 486-493, 1976.

[42] S. Raboni, S. Bettati, and A. Mozzarelli, “Tryptophan synthase: a mine for enzymologists," Cellular and Molecular Life Sciences, vol. 66, no. 14, pp. 2391-2403, 2009.

[43] S. P. Nair, J. L. Harwood, and R. A. John, "Direct identification and quantification of the cofactor in glutamate semialdehyde aminotransferase from pea leaves," FEBS Letters, vol. 283, no. 1, pp. 4-6, 1991.

[44] S. Brody, J. S. Andersen, C. G. Kannangara, M. Meldgaard, P. Roepstorff, and D. Von Wettstein, "Characterization of the different spectral forms of glutamate 1-semialdehyde aminotransferase by mass spectrometry," Biochemistry, vol. 34, no. 49, pp. 15918-15924, 1995.

[45] G. Fenalti, R. H. P. Law, A. M. Buckle et al., "GABA production by glutamic acid decarboxylase is regulated by a dynamic catalytic loop," Nature Structural and Molecular Biology, vol. 14, no. 4, pp. 280-286, 2007.

[46] M. Bertoldi, M. Gonsalvi, R. Contestabile, and C. B. Voltattorni, "Mutation of tyrosine 332 to phenylalanine converts 
dopa decarboxylase into a decarboxylation-dependent oxidative deaminase," Journal of Biological Chemistry, vol. 277, no. 39, pp. 36357-36362, 2002.

[47] P. Burkhard, C.-H. Tai, C. M. Ristroph, P. F. Cook, and J. N. Jansonius, "Ligand binding induces a large conformational change in $O$-acetylserine sulfhydrylase from Salmonella typhimurium," Journal of Molecular Biology, vol. 291, no. 4, pp. 941-953, 1999.

[48] H. Tian, R. Guan, E. Salsi et al., "Identification of the structural determinants for the stability of substrate and aminoacrylate external schiff bases in $\mathrm{O}$-acetylserine sulfhydrylase-A," Biochemistry, vol. 49, no. 29, pp. 6093-6103, 2010.

[49] A. Mozzarelli, S. Bettati, B. Campanini et al., "The multifaceted pyridoxal $5^{\prime}$-phosphate-dependent $O$-acetylserine sulfhydrylase," Biochimica et Biophysica Acta, vol. 1814, no. 11, pp. 14971510, 2011.

[50] S. Raboni, R. Contestabile, F. Spyrakis et al., "Pyridoxal 5'phosphate-dependent enzymes: catalysis, conformation and genomics," in Comprehensive Natural Products II Chemistry and Biochemistry, El Sevier, Oxford, UK, 2010. 

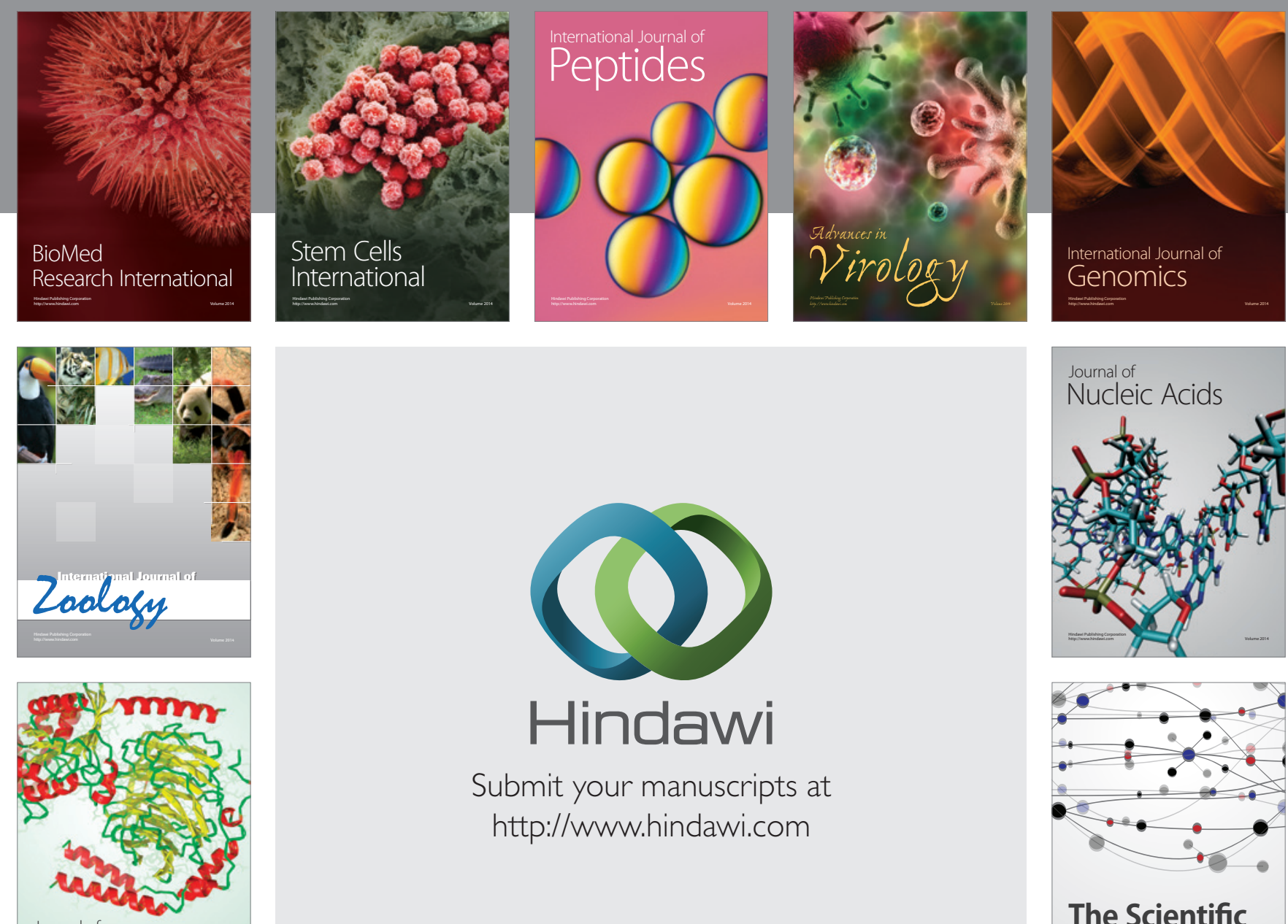

Submit your manuscripts at

http://www.hindawi.com

Journal of
Signal Transduction
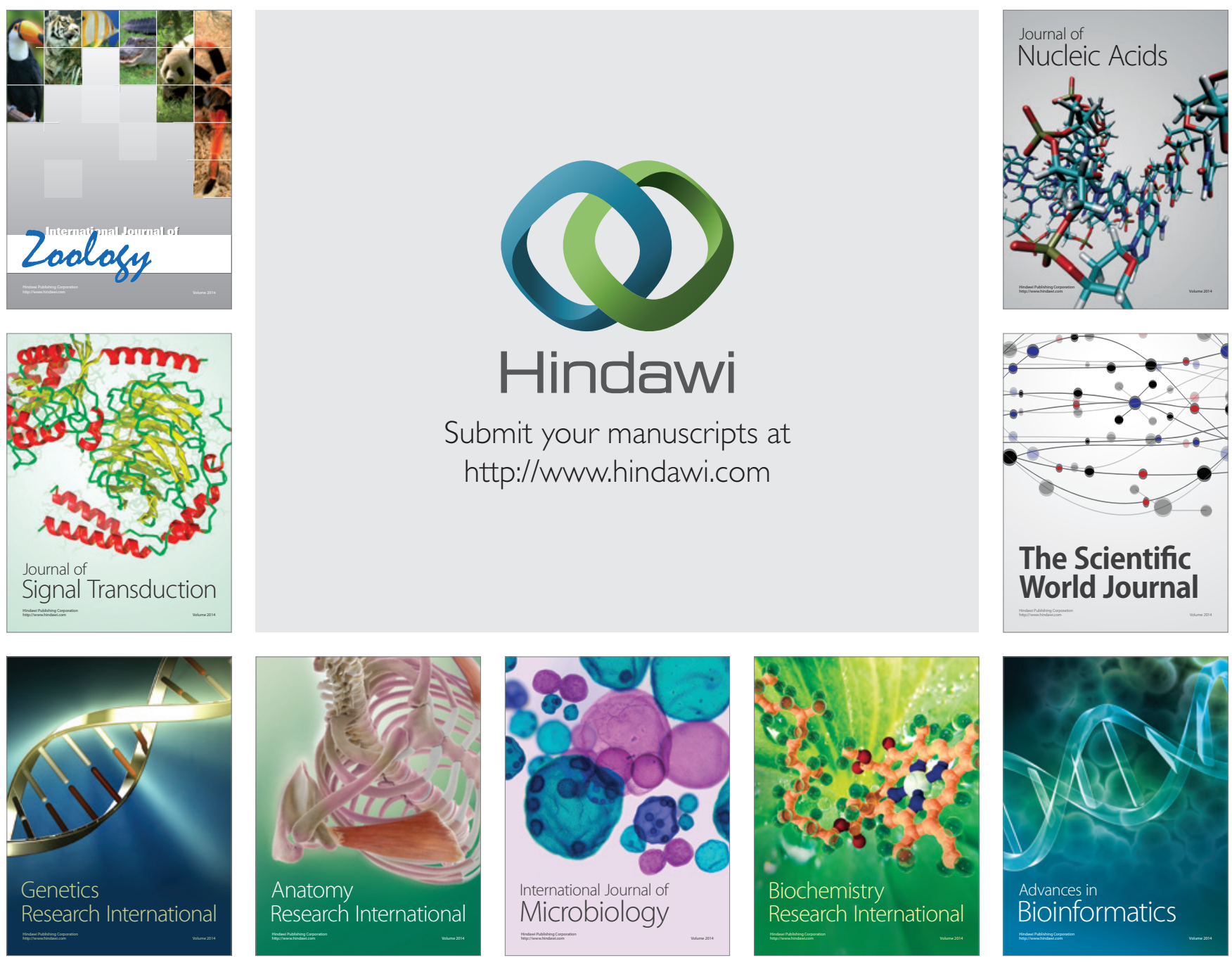

The Scientific World Journal
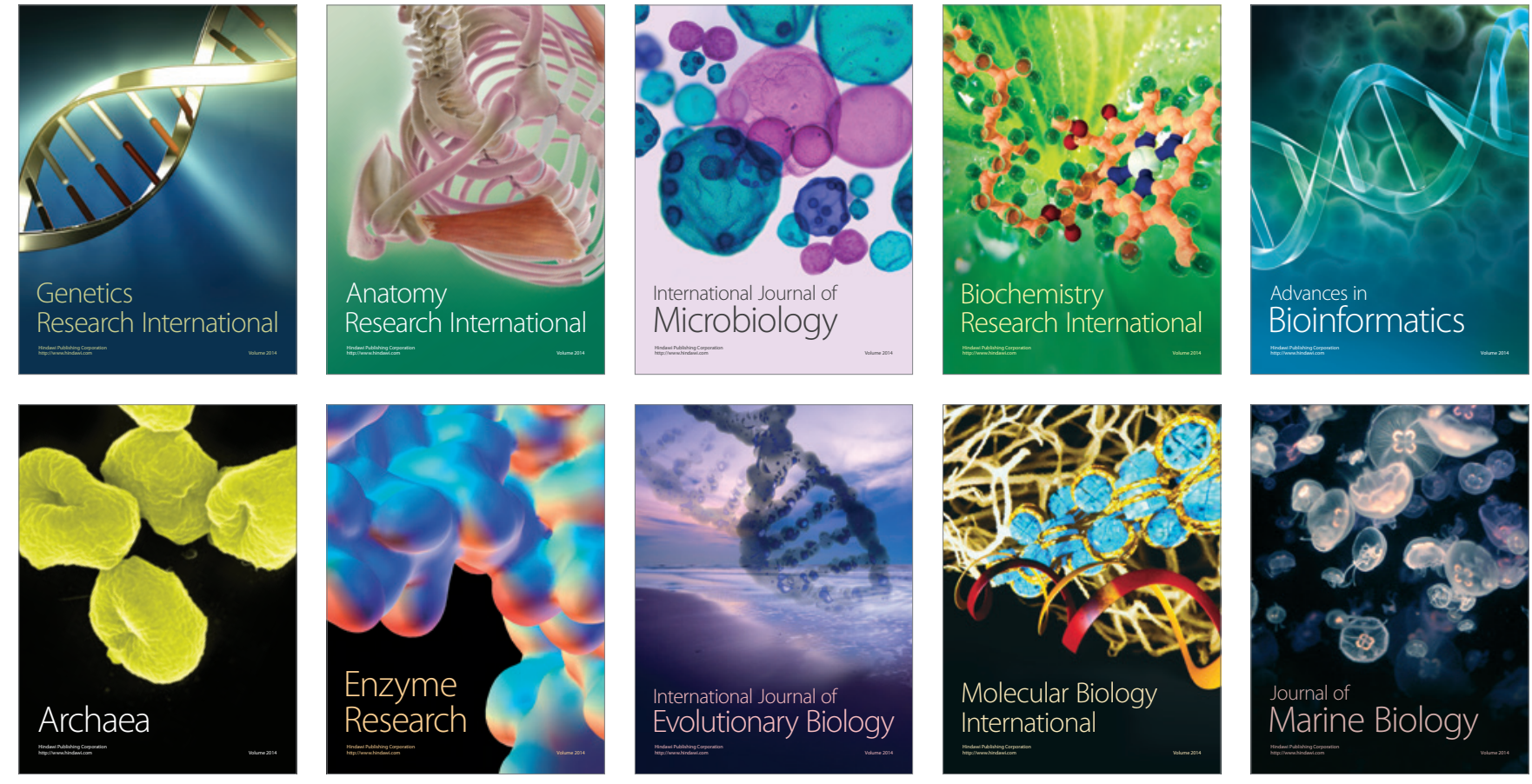